\title{
Estimación de la volatilidad de los precios de las acciones de la BMV mediante el modelo CARR El caso de AMX-L*
}

Fernando $\mathrm{H}$.

Servín y Silva

Universidad de Celaya. fservin@udec.edu.mx

*Se agradece a los evaluadores sus importantes comentarios que han hecho de este artículo uno mejor.

\section{Resumen}

Este artículo tiene como propósito presentar la estimación del Modelo Autorregresivo de Rango Condicional (CARR) $(\mathrm{p}, \mathrm{q})$ a partir de tres procedimientos distintos: como un modelo ARMA $(p, q)$ empleando estimadores Mínimo-Cuadráticos (MCO), como un modelo GARCH $(\mathrm{p}, \mathrm{q})$ empleando estimadores de Cuasi-Máxima Verosimilitud (QMLE) y como un modelo $\operatorname{GARCHX}(\mathrm{p}, \mathrm{q})$ usando estimadores de Máxima Verosimilitud (MLE). La aplicación de estos tres procedimientos está en función de las consideraciones que se hacen sobre la distribución de probabilidad de los errores y de las propiedades de los estimadores que se quieren resaltar. En una segunda etapa, en lugar de emplear series con observaciones diarias, se hacen estimaciones de los mismos modelos empleando observaciones semanales, quincenales y mensuales. Al final mostramos que los mejores resultados se obtienen mediante los modelos $\operatorname{ARMA}(p, q)$ y GARCHX (p, q), independientemente de que los rangos de valores extremos se obtengan a partir de datos diarios, semanales o mensuales.

Palabras clave: volatilidad, rango, modelos CARR, estimadores MLE, estimadores QMLE. 


\title{
Volatility estimation of Mexican Equity Shares based on the CARR Model: The AMX-L Case
}

\begin{abstract}
This article aims to estimate the Conditional Autoregressive Range Model CARR (p, q) by the use of three different procedures: The ARMA model (p, q) using least squares estimators (MCO), the GARCH model $(\mathrm{p}, \mathrm{q})$ using Quasi-Maximum Likelihood Estimators (QMLE) and the GARCHX model (p, q) using Maximum Likelihood Estimators (MLE). The usage of these three procedures is in function of the considerations made on the probability distribution of errors and the estimators' properties that are to be emphasized. At a second stage, instead of daily observations, estimations of the same models were made on a, weekly, fortnightly, and monthly observation basis. Finally we demonstrate that the best results are obtained through the $\operatorname{ARMA}(\mathrm{p}, \mathrm{q})$ and $\operatorname{GARCHX}$ models $(\mathrm{p}, \mathrm{q})$, regardless of whether the extreme value ranges come from daily, weekly, fortnightly or monthly observations.
\end{abstract}

Keywords: volatility, range, CARR models, (MLE) estimators, (QMLE) estimators.

\section{Introducción}

Este trabajo es una aplicación del artículo de Chou (2005) en el que se presenta el rango como una forma alternativa de medir la volatilidad de los precios de las acciones de la Bolsa Mexicana de Valores. La discusión se desarrolla a partir de la perspectiva econométrica propuesta por Chou, por lo cual se hace la presentación y posterior utilización del Modelo Autorregresivo de Rango Condicional (CARR).

El punto de partida de estos modelos se encuentra en los trabajos de Engle (2002) cuando da a conocer una extensión de la metodología GARCH al análisis de modelos de error multiplicativo. Este autor presenta esquemas tales como el autorregresivo de duración condicional ACD y el autorregresivo de rangos condicionales CARR. Posteriormente, Chou (2005) publica un artículo que dedica exclusivamente al análisis y obtención de un modelo CARR (p, q) para estimar la volatilidad del índice S\&P500.

La bibliografía consultada para la realización de este trabajo incluye, entre otros títulos, Chou y Wang (2005), quienes de una manera sintética presentan los rasgos característicos del CARR; Magdoni y Atiya (2003), quienes hacen estimaciones 
del rango mediante los precios máximo y mínimo de la acción de IBM; Alizadeh, Brandt y Diebold (1999), quienes resaltan la utilidad práctica de los estimadores QMLE para la determinación de este tipo de modelos.

El supuesto de normalidad de los errores, del que inicialmente parten los procedimientos de la famila GARCH, con frecuencia se ve quebrantado al incorporar modelos tales como el CARR o el ACD. Las trascendentales aportaciones de Woolridge y Bollerslev (1992), con sus procedimientos de cuasi-máxima verosimilitud, permiten que los estimadores sean consistentes, independientemente de que la distribución de los errores no sea una normal.

En este artículo se obtiene el modelo CARR por tres procedimientos distintos, de esta manera se analiza la sensibilidad que tienen los parámetros estimados ante los diferentes supuestos que se hacen en cuanto a la forma de la distribución de probabilidad de los errores. Se parte del supuesto clásico de Normalidad $\mathrm{N}(0,1)$, se sigue con el de la Distribución Exponencial Unitaria y se concluye con el de los Errores Generalizados (DEG). Más específicamente se determina primero el modelo CARR usando la metodología Box \& Jenkins con estimadores MCO; posteriormente, y con los mismos datos, se obtiene el modelo CARR a partir del esquema GARCH empleando QMLE y asumiendo que la distribución de los errores es una exponencial unitaria tal como lo propone en su artículo Chou (2005); finalmente, se construye un modelo CARRX asumiendo normalidad asintótica, empleando MLE y tomando la Distribución de Errores Generalizados.

Una alternativa más de estimación que se puede emplear es el Método Generalizado de los Momentos (GMM) propuesto por Rich (1991). Si bien es cierto que este procedimiento muestra algunas ventajas de carácter práctico (no requerir el conocimiento exacto de la función de distribución de los errores), presenta una importante desventaja: los estimadores GMM son ineficientes en relación con la máxima verosimilitud cuando la verdadera función de distribución de los errores es efectivamente una normal.

El empleo de una función generalizada de error o la t-student, propuestas por Nelson (1991), ofrecen una mejor alternativa, pues se demuestra que la ineficiencia relativa de usar una normal cuando la verdadera distribución es una t-student es muy pequeña, tal como lo comprueba De Arce (2004). 
Contar con una familia de distribuciones exponenciales con la suficiente flexibilidad en cuanto a la asignación de los parámetros, que permitan crear condiciones particulares de sesgo y curtosis, se convierte en la opción de estimación más práctica para este tipo de modelos. Ésta es la razón por la que en el presente documento se opta por la Función Generalizada de Error en lugar del Método Generalizado de Momentos. En la segunda parte de este artículo se dan las definiciones pertinentes: rendimiento, volatilidad cierre/cierre, volatilidad máximo/mínimo, etc., así como un presentación de las propiedades empíricas que muestra las series de tiempo de los rangos de valores extremos. En la tercera parte se hace la presentación del modelo CARR, así como de la forma de estimación mediante los procedimientos antes mencionados. En la cuarta parte se describen los datos que se emplean en la estimación de los modelos. En la quinta parte se presentan los modelos obtenidos; además, se incluyen las estimaciones a partir de las series de rangos semanales, quincenales y mensuales, con el propósito de identificar similitudes y diferencias en los resultados en relación con la separación entre los datos. En la parte final se presentan las conclusiones, la bibliografía y los apéndices.

\section{Antecedentes}

Precios, rendimientos y diversas medidas de volatilidad

El rendimiento de cualquier activo financiero está ligado al tiempo de posesión del mismo. La manera más común de calcularlo es mediante:

$$
\mathrm{R}_{\mathrm{t}}=\frac{\mathrm{P}_{\mathrm{t}}-\mathrm{P}_{\mathrm{t}-1}}{\mathrm{P}_{\mathrm{t}-1}}
$$

El rendimiento mide la variación de los precios con respecto al tiempo; ello significa que la serie de los rendimientos diarios, semanales o mensuales dan la volatilidad de los precios -usualmente de cierre- con respecto al día, semana o mes inmediato anterior.

De acuerdo con Ruey "una característica fundamental de la volatilidad de los precios es que no es directamente observable" (2005, p 98). La volatilidad diaria, cuando se mide a partir de las variaciones en la serie de los rendimientos, no puede ser observada directamente porque se basa en solo un precio del día. Si por el contrario se toman dos o más observaciones del intradía, entonces se pueden medir las diferencias que van teniendo estos en periodos de diez minutos, media hora o de toda la jornada. 
De las diversas formas que existen para medir la volatilidad aquí sólo se hace referencia a dos de ellas: la de Parkinson (que usa los valores máximo/mínimo de la jornada) y la de cierrre/cierre (que usa la variación entre los precios de cierre de dos sesiones consecutivas). Sin embargo, existen otras alternativas tales como la de German and Klass (se calcula a partir de los precios de apertura/máximo/mínimo/cierre), la de Yang Zhang y otras. En todos los procedimientos existentes, a excepción de la de cierre/cierre, se emplean dos o más observaciones de la jornada para su determinación y en todos los casos esos estimadores son más eficientes que el que se usa para la volatilidad cierre/cierre, de acuerdo con Malik y Amir (2003).

\section{Variación entre días consecutivos vs. variación intradía}

Las gráficas de precios máximos/mínimos/cierre son de uso común en la representación de series de precios de activos financieros. Estos diagramas tienen la ventaja de lograr una esquematización muy cercana a lo que sucede en la realidad con las variaciones de los precios de las acciones. A su vez, el rango del día es un número no negativo asociado a la longitud de cada una de las barras. Una sucesión de barras correspondiente a un número determinado de días de negociación da una visión en el tiempo del comportamiento de los precios, así como de la volatilidad que ha venido presentando el título.

El rango, a diferencia de la desviación estándar, es más congruente al momento de explicar la volatilidad diaria en términos de la volatilidad intradía, pues en esencia se está hablando de lo mismo. Tanto para la variación diaria como para el intradía, la volatilidad correspondiente a la jornada es la diferencia entre el precio máximo menos el mínimo que alcanza el título durante la sesión. Esta congruencia no se logra al emplear como mediada de la volatilidad la desviación estándar de los rendimientos, ya que para la volatilidad diaria se hacen unas consideraciones y para la intradía otras. Esto se puede explicar de la siguiente forma: en el primero de los casos se trata de una serie igualmente espaciada y en el segundo es irregularmente espaciada. Esto nos conduce al hecho de que estadísticamente se tienen que calcular de manera distinta y, por tanto, ambas no son numéricamente equivalentes como en el caso del rango. La congruencia y consistencia son propiedades fundamentales de cualquier desarrollo científico, sin importar el área de conocimiento del que se trate. 


\section{La volatilidad de valores extremos y sus propiedades}

Parkinson (1980) propone una expresión similar a la que se presenta en (1) sólo que se usa para medir la volatilidad con el precio máximo y mínimo de la sesión.

$$
\mathrm{RP}_{\mathrm{t}}=\operatorname{Ln}\left(\mathrm{rp} \mathrm{t}_{\mathrm{t}}+1\right)=\operatorname{Ln}\left(\frac{\text { PMáx }_{\mathrm{t}}}{\text { PMín }_{\mathrm{t}}}\right)
$$

La serie de rangos de Parkinson muestra rasgos característicos que también se observan en las series de rendimientos cierre/cierre; por ejemplo, la volatilidad es predecible independientemente que ésta se mida a través del rango o de la desviación estándar. Lo que sí puede cambiar son las propiedades de los estimadores que se emplean para uno y otro tipo de secuencia y esto dependerá de cada situación en particular.

Otras características empíricas que se mencionan a continuación, y que serán discutidas en páginas posteriores, son las siguientes:

A. La distribución de frecuencias de los rangos $\mathrm{RP}_{\mathrm{t}} \geq 0$ para $\mathrm{t}=1,2,3, \ldots$, etc., se encuentra en el primer cuadrante, es acampanada, asimétrica y con exceso de curtosis.

B. La secuencia de valores $\left\{\mathrm{RP}_{\mathrm{t}}\right\}$ es igualmente espaciada. De los trabajos consultados algunos toman el rango con observaciones diarias; otros con observaciones semanales, quincenales, etc. En la mayoría de los artículos se determina la volatilidad de índices (S\&P 500, FETSE 100), pero hay otros que se refieren a los precios de acciones.

C. Como se verifica más adelante, la serie de los rangos $\left\{\mathrm{RP}_{\mathrm{t}}\right\}$ es estacionaria. Este hecho permite obtener estimaciones de $\mathrm{RP}_{\mathrm{t}+1}$ con la metodología ARMA.

D. Otros rasgos característicos de $\left\{\mathrm{RP}_{\mathrm{t}}\right\}$ son agrupamiento de la volatilidad, reversión a la media y persistencia. 


\section{El modelo CARR}

\section{Modelo autorregresivo de rangos condicionales}

Este modelo, una adecuación del GARCH, permite estimar el rango condicional del día t a partir de la información disponible hasta el día inmediato anterior $\left(\mathrm{I}_{\mathrm{t}-1}\right)$.

Sea $Y_{t}=\left(R P_{t} \mid I_{t-1}\right)$ el rango esperado condicional con información disponible hasta el día $\mathrm{t}$ - 1 y $\mathrm{RP}_{\mathrm{t}}$ el rango observado del día t, entonces se puede plantear el modelo $\mathrm{RP}_{\mathrm{t}}=\varepsilon_{\mathrm{t}} \mathrm{Y}_{\mathrm{t}}$ similar al ACD propuesto por Engle y Russell (1998), perteneciente a la familia de modelos con error multiplicativo.

El rango de Parkinson del día t es una adecuación del rango condicional esperado $\mathrm{Y}_{\mathrm{t}}$ corregido por un error estocástico $\varepsilon_{\mathrm{t}}$. A su vez, el rango esperado condicional se expresa mediante la suma de un término constante $(\omega)$, una ponderación del rango esperado condicional de periodos anteriores $\beta_{\mathrm{j}} \mathrm{Y}_{\mathrm{t}-\mathrm{j}}$ más una ponderación del rango observado de periodos inmediatos anteriores $\alpha_{j} R_{t-j}$.

$$
\begin{gathered}
R P_{t}=Y_{t} \varepsilon_{t} \\
Y_{t}=\omega+\sum_{i=1}^{p} \alpha_{i} R P_{t-i}+\sum_{j=1}^{q} \beta_{j} Y_{t-j}
\end{gathered}
$$

Sin perder generalidad se puede suponer que los $\varepsilon_{\mathrm{t}}$ son iid. Además, $\varepsilon_{\mathrm{t}}>0$ para todo t y $\varepsilon_{\mathrm{t}}$ se distribuye siguiendo una función de densidad $\mathrm{f}$ (.) con la propiedad de que $\mathrm{E}\left[(\mathrm{f}()]=\right.$.1 . Como condición de suficiencia se pide que los parámetros $\omega, \alpha_{\mathrm{j}} \mathrm{y} \beta_{\mathrm{j}}$ deben ser todos positivos y así tener la certeza de que Yt $>0$. Si se emplea MLE la función de verosimilitud estará dada por:

$$
L\left(\alpha_{i}, \beta_{i}, R P_{1}, \ldots, R P_{n}\right)=-\sum_{t=1}^{n}\left[\ln \left(Y_{t}\right)+\frac{R P_{t}}{Y_{t}}\right]
$$

Los modelos de error multiplicativo se formulan como un ARMA (máx. (p, q), q). Si se sustituye $\eta_{t} \equiv R P_{t}-Y_{t}$ queda la ecuación: 


$$
R P_{t}=\omega+\sum_{j=1}^{\max (m, q)}\left(\alpha_{j}+\beta_{j}\right) R P_{t-j}-\sum_{j=i}^{q} \beta_{j} \eta_{t-j}+\eta_{j}
$$

Para una estimación válida del ARMA es necesario que se cumplan una serie de condiciones: los residuos del modelo obtenido deben ser compatibles con ruido blanco y los coeficientes deben satisfacer las condiciones de estacionariedad e invertibilidad.

$\mathrm{Si}_{\mathrm{RP}}$ es un proceso estacionario, el valor esperado no condicional del rango estará dado por la expresión:

$$
E\left(Y_{t}\right)=\frac{\omega}{1-\sum_{i=1}^{p} \alpha_{i}+\sum_{j=1}^{q} \beta_{j}}
$$

Naturalmente se requiere que:

$$
\sum_{i=1}^{p} \alpha_{i}+\sum_{j=1}^{q} \beta_{j}<1
$$

Los modelos ARMA se pueden estimar mediante MCO o mediante MLE. En última instancia, las propiedades que se obtienen para el modelo, así como los resultados alcanzados, son equivalentes independientemente de que se haya utilizado uno u otro procedimiento.

Si los errores $\varepsilon_{\mathrm{t}}$ no son iid entonces se puede asignar una distribución de probabilidad no negativa, con media igual a la unidad y con varianza dependiente del tiempo. Entre las opciones que se mencionan se puede contemplar a la exponencial.

Desde esta nueva condición para los $\varepsilon_{\mathrm{t}}$, la estimación del modelo CARR se logra mediante estimadores de cuasi-máxima verosimilitud, tal como lo propone Engle y Russell (1998). Se trata de generar un modelo GARCH para la raíz cuadrada del rango de valores extremos sin incluir el término constante en la ecuación de la media. Naturalmente, el modelo GARCH alcanzado debe cumplir con una serie de condiciones: 
A. Los coeficientes de la ecuación del rango esperado condicional no necesariamente deben ser estadísticamente significativos, pero sí se requiere que $\omega>0, \alpha_{j} \geq 0$ y $\beta_{j} \geq 0$.

B. Los residuos estandarizados no deben estar serialmente correlacionados ni presentar el efecto ARCH.

C. Con los coeficientes de la ecuación obtenida se pueden generar las expresiones (6) y (7) con su respectiva interpretación de acuerdo con el contexto.

D. Los estimadores QMLE son consistentes, aunque no necesariamente eficientes.

E. Si se demuestra que la distribución de los errores $\left\{\varepsilon_{\mathrm{i}}\right\}$ coincide con la que se supuso (exponencial), entonces los estimadores QMLE también son eficientes.

El modelo presentado en (3) se puede generalizar agregando variables explicativas $\mathrm{x}_{\mathrm{k}} \operatorname{con} \mathrm{k}=1,2, \ldots$ etc.

$$
\begin{gathered}
\mathrm{RP}_{\mathrm{t}}=\mathrm{Y}_{\mathrm{t}} \varepsilon_{\mathrm{t}} \\
\mathrm{Y}_{\mathrm{t}}=\omega+\sum_{\mathrm{i}=1}^{\mathrm{p}} \alpha_{\mathrm{i}} \mathrm{RP} \mathrm{P}_{\mathrm{t}-\mathrm{i}}+\sum_{\mathrm{j}=1}^{\mathrm{q}} \beta_{\mathrm{j}} \mathrm{Y}_{\mathrm{t}-\mathrm{j}}+\sum_{\mathrm{l}=1}^{\mathrm{k}} \xi_{1} \mathrm{X}_{\mathrm{t}-1}
\end{gathered}
$$

Las ecuaciones de (8) no son otra cosa más que un GARCHX. Esta especificación aplicada a la serie de los rangos $\mathrm{RP}_{\mathrm{t}}$, y que incluye variables exógenas como explicativas, se conoce con el nombre de CARRX (p, q) y la estimación de la volatilidad esperada condicional se puede obtener mediante un ARMAX.

La estimación del modelo CARRX también se puede lograr mediante un GARCHX empleando un procedimiento similar al anterior. El modelo multiplicativo con al menos una variable exógena puede también ser estimado a partir de la raíz cuadrada de la serie RPt con media cero y suponiendo que la distribución de $\operatorname{los}\left\{\varepsilon_{\mathrm{i}}\right\}$ es asintóticamente normal y con variable exógena $\mathrm{x}_{\mathrm{i}}$. Dadas estas nuevas condiciones es posible emplear MLE. 
En este trabajo se utiliza la Distribución de Error Generalizado (GED) que es una familia de distribuciones exponenciales que incluyen a la normal. El dominio de la función de densidad de probabilidad x está definida dentro del intervalo x $€(-\infty$, $\infty)$ y la distribución es especificada por tres parámetros: $\mu \in(-\infty, \infty)$, el cual ubica a la moda (que es igual a la media y a la mediana) de la distribución, $\sigma \in(-\infty, \infty)$ que define la dispersión y $\delta \in(0, \infty)$ que controla el sesgo.

$$
\mathrm{f}(\mu, \sigma, \delta)=\frac{\delta}{2 \sigma \Gamma(1 / \delta)} \mathrm{e}^{-(|x-\mu| / \sigma)^{\delta}}
$$

Si $\delta=2$ se trata de una distribución normal con media $\mu$ y desviación estándar $\sigma / 2$. En la medida en que $\delta \rightarrow 1$ la distribución tiene cada vez más curtosis y si $\delta$ $=1$ entonces es una doble exponencial o distribución de Laplace. En el límite, si $\delta \rightarrow 0$ entonces se tiene una distribución uniforme (prácticamente paralela al eje horizontal). La función de máxima verosimilitud (MLE) asociada a esta familia de distribuciones de error queda representada mediante la siguiente expresión:

$$
\mathrm{L}_{\mathrm{t}}=-\frac{1}{2} \ln \left(\frac{\Gamma(1 / \delta)^{3}}{\Gamma(3 / \delta)(\delta / 2)^{2}}\right)-\frac{1}{2} \ln \sigma_{\mathrm{t}}^{2}-\left(\frac{\Gamma(3 / \delta)\left(\mathrm{y}_{\mathrm{t}}-\mathrm{X}_{\mathrm{t}}^{\prime} \vartheta\right)^{2}}{\sigma^{2} \Gamma(1 / \delta)}\right)
$$

\section{Datos y metodología}

\section{De los datos empleados}

En este inciso se describen las características de la secuencia de los datos, así como de los algoritmos empleados en la determinación de los modelos. Se incluyen 4108 observaciones correspondientes a los precios máximos y mínimos de la acción AMX Serie L generados durante 2054 días que van del 7/02/2001 al 3/04/2009 y que fueron obtenidos de las bases de datos de Economática. También se determinó el rango de valores extremos semanal, quincenal y mensual. En cada caso, se toma el logaritmo natural del máximo entre el mínimo del día, de toda la semana, de la quincena o de todo el mes.

El primer y tercer modelo se estimó mediante técnicas de regresión no lineal; en ambos casos se uso el algoritmo de mínimos cuadrados no lineales Marquardt. Las 
estimaciones con mínimos cuadrados no lineales son asintóticamente equivalentes a las que se obtienen mediante máxima verosimilitud. El segundo modelo se obtuvo mediante estimadores robustos de cuasi-máxima verosimilitud de acuerdo con el procedimiento propuesto por Bollerslev-Woldridge.

\section{Figura 1}

\section{Gráfica de línea del rango alto/bajo}

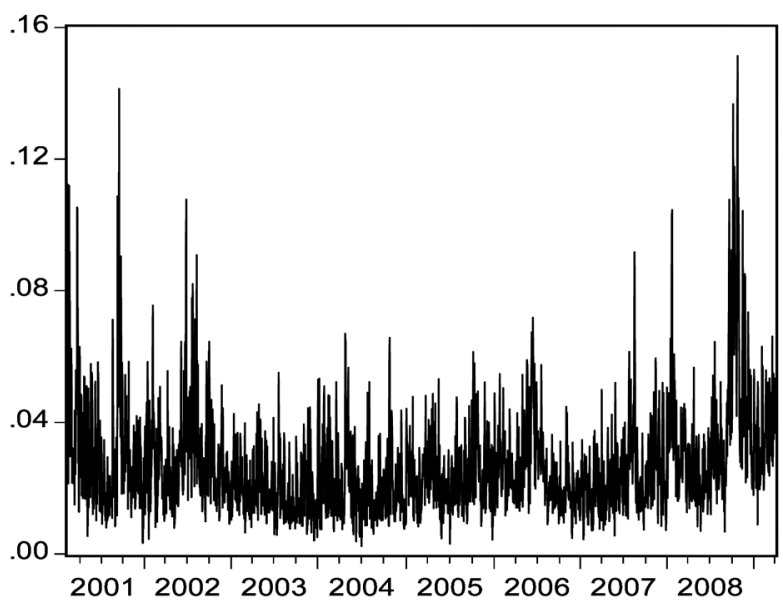

La figura 1 esquematiza la serie de las volatilidades diarias; a partir de esta gráfica se observa que se intercalan periodos de estabilidad con diferencias mínimas en la variabilidad de los precios y, posteriormente, le siguen periodos de inestabilidad con pronunciadas fluctuaciones. Aunque un poco más difícil de identificar, también se observa la presencia de cierto nivel de ciclicidad. El agrupamiento de la volatilidad tiene otras implicaciones tales como la persistencia y la reversión a la media, las cuales serán discutidas más adelante.

Desde un punto de vista financiero, el agrupamiento de la volatilidad implica que los shocks en la volatilidad de hoy pueden influir en las expectativas de volatilidad de muchos periodos adelante. La volatilidad será más o menos persistente en la medida en que los efectos de la llegada de nueva información persistan por mayor o menor tiempo después de su llegada. La persistencia en la serie de los rendimientos será mayor en la medida en que la historia de la serie sea la única depositaria de la memoria del mercado. En la medida en que se cuente con modelos autorregresivos multivariados o con modelos univariados que incluyan otras variables explicativas, ésta se distribuirá entre los diversos términos que describen el fenómeno. 
La volatilidad será muy persistente en la medida en que la sumatoria que aparece en (7) sea muy próxima a la unidad.

Por su parte, la reversión a la media establece que hay un nivel normal de volatilidad al que la serie deberá regresar. Al hacer pronósticos de muy largo plazo, dicha volatilidad debe converger a un nivel constante. Ese valor es la volatilidad esperada no condicional y está determinado por la expresión (6).

En la figura 2 se aprecia la distribución de frecuencia de las volatilidades alto/bajo. Para la serie de los rangos diarios se observa que es asimétrica con media .0269, coeficiente de sesgo 2.218 y coeficiente de curtosis de 11.42 . Tiene la forma de una distribución lognormal, tal como se demuestra más adelante (figura 4).

\section{Figura 2}

\section{Distribución de frecuencia de los rangos}
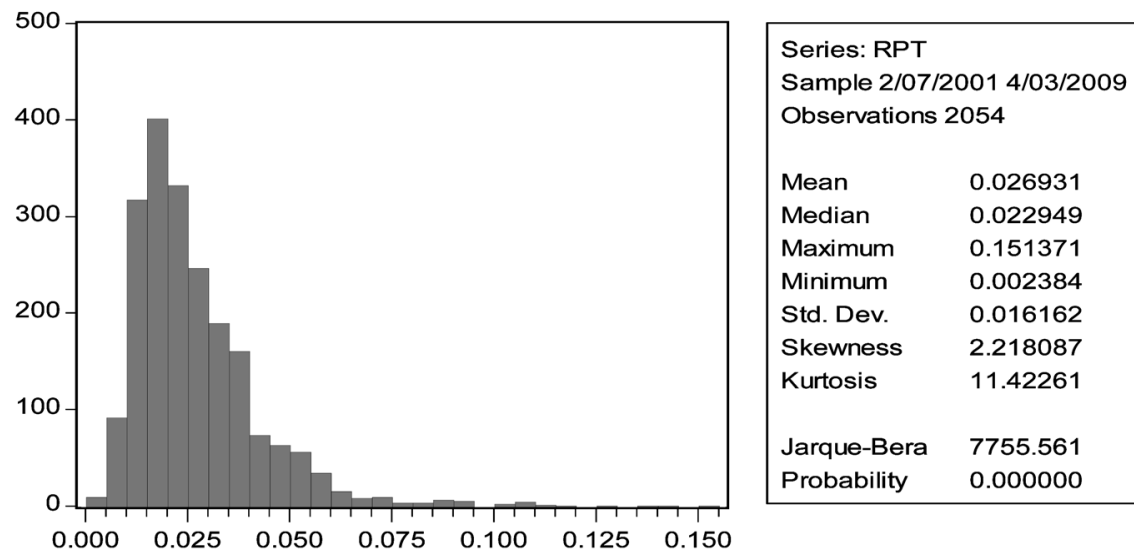

El patrón de autocorrelación y autocorrelación parcial de la serie $\left\{\mathrm{RP}_{\mathrm{t}}\right\}$ indica que ambas tienen un comportamiento en el que van decayendo de forma gradual a medida que aumenta el número de rezagos. Estos patrones conducen a la idea de que es posible ajustar un modelo autorregresivo que en principio puede ser de la forma ARMA (p, q). Para corroborar esta posibilidad se hace la prueba de la raíz unitaria de Phillips-Perron y se verifica que la serie es estacionaria (cuadro 3). 


\section{Resultados}

\section{Primer modelo ajustado}

Se estima mediante mínimos cuadrados como un modelo ARMA $(1,1)$ y es uno de los que mejor se ajusta a los datos.

$$
\begin{gathered}
R P_{t}=Y_{t} \varepsilon_{t} \\
Y_{t}=.0267+.226 R P_{t-1}+.720 Y_{t-1}
\end{gathered}
$$

Los tres coeficientes, además, son positivos y el proceso autorregresivo es estacionario en covarianza e invertible, tal como se aprecia en el cuadro 4. En relación con la persistencia se puede decir que $.226+.720=.946$ y el valor esperado del rango no condicional esta dado por $\mathrm{E}(\mathrm{Y})=.028$.

El diagrama de los rangos esperados condicionales obtenidos mediante el modelo (en gris) y los rangos observados (en negro) aparece en la figura 3. Dado el cumplimiento de las condiciones antes descritas, se puede decir que los estimadores mínimos cuadrados tienen distribuciones asintóticamente normales y los estadísticos $t$ de student y F son asintóticamente válidos.

Figura 3

Gráfica de línea de valores observados (RPt) y estimados (Yt)

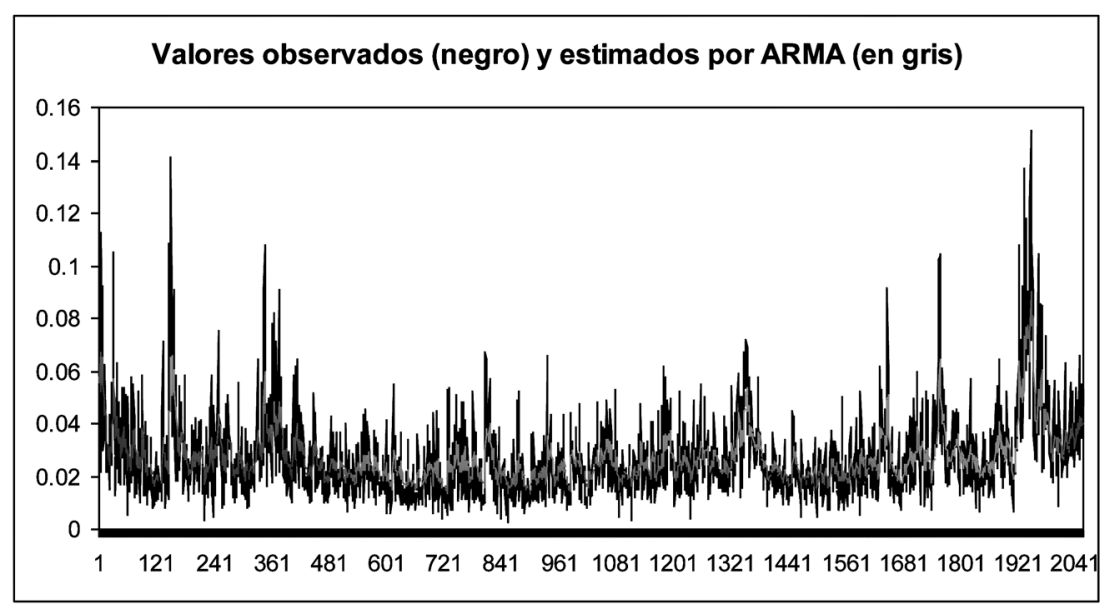




\section{El segundo modelo}

Después de corroborar que los errores $\left\{\varepsilon_{\mathrm{i}}\right\}$ no son iid (ver cuadro 5), se puede suponer que éstos siguen una distribución no negativa con media unitaria y varianza cambiando en el tiempo. Entre las distribuciones propuestas, se menciona a la exponencial unitaria, tal como lo sugiere Chou (2005).

Para obtener una estimación consistente de los parámetros se emplea el método de cuasi-máxima verosimilitud y así lograr un modelo GARCH $(1,1)$ a partir de la raíz cuadrada de la serie de los rangos $\mathrm{RP}_{\mathrm{t}}$ y suprimiendo el término constante de la ecuación de la media (cuadro 6).

$$
\begin{gathered}
R P_{t}=Y_{t} \varepsilon_{t} \\
Y_{t}=.0017+.203 R P_{t-1}+.731 Y_{t-1}
\end{gathered}
$$

Es importante resaltar que el valor constante $\mathrm{c}=.0017$ es el que corresponde al término constante de la ecuación del rango medio condicional y no al término constante de la ecuación de la media del modelo GARCH.

Este modelo satisface todas las restricciones incluyendo las de los signos. El rango esperado condicional tiene una parte constante de .0017 más la contribución que hace la nueva información contenida en los dos términos correspondientes al periodo inmediato anterior. En este caso, el coeficiente $\alpha_{1}=.203$ significa que la información contenida en el rango inmediato anterior produce un impacto significativamente menor con respecto a $\beta_{1}=.731$, que es el impacto de largo plazo correspondiente al rango esperado condicional. En relación con la persistencia se puede decir que $.203+.731=.934$ y el valor esperado del rango no condicional $\mathrm{E}$ $(\mathrm{Y})=.0257$.

La validación de este tipo de modelos requiere que los errores estandarizados no estén autocorrelacionados y que tampoco se encuentre presente el efecto ARCH. Ambas condiciones son satisfechas y lo único que no se cumple es que los errores sigan una distribución exponencial unitaria. Sin embargo, este último punto impacta en la eficiencia de los estimadores QMLE, pero no en su consistencia (cuadro 6). 


\section{Tercer modelo (CARRX)}

Este modelo incluye como variable explicativa adicional la cantidad de títulos negociados (Cant Tit.) de la acción AMX, L correspondiente a cada uno de los días incluidos en la serie original de datos.

De acuerdo con Baklaci y Kasman, "Tal como fue inicialmente sugerido por Morgan (1976), el volumen es considerado como el factor de riesgo que tiene una mayor contribución a la volatilidad de los rendimientos, particularmente en los mercados menos líquidos como es el caso de los emergentes" (2006, p. 116)

En la estimación de este modelo se usan mínimos cuadrados no lineales partiendo del supuesto de una distribución asintóticamente normal para los errores. Esto permite emplear la Distribución de Error Generalizado (GED), lo cual da la libertad de que se adecue la distribución para los errores de acuerdo con los valores asignados para la media $(\mu)$, la desviación estándar $(\sigma)$ y el coeficiente de sesgo $(\delta)$.

Los resultados dan un valor $\delta=2$, el cual es significativo y corresponde a una distribución normal. A su vez $\omega=.0269, \alpha=.15$ y $\beta=.60$, todos ellos positivos y estadísticamente significativos.

El coeficiente de la variable exógena Cant. Tit, no obstante que es muy pequeño $\left(-2.50\right.$ por $\left.10^{-10}\right)$, es estadísticamente significativo y resulta con signo menos. El hecho de que sea un número tan pequeño es perfectamente congruente con el modelo, pues la variable Cat. Tit, toma valores muy grandes (AMX, L concreta en promedio operaciones por 36 millones $\left(36\right.$ por $\left.10^{6}\right)$ de títulos intercambiados diariamente). De igual manera, que este coeficiente tenga signo negativo sugiere que hay una relación inversa entre la volatilidad que presenta el título y su bursatilidad (ver cuadro 7).

$$
\begin{gathered}
\mathrm{RP}_{\mathrm{t}}=\mathrm{Y}_{\mathrm{t}} \varepsilon_{\mathrm{t}} \\
\mathrm{Y}_{\mathrm{t}}=.0269+.15 \mathrm{RP}_{\mathrm{t}-1}+.60 \mathrm{Y}_{\mathrm{t}-1}-.00000000025 \mathrm{Cant} . \text { Tit }
\end{gathered}
$$

La volatilidad media condicional del día de hoy está determinada por cuatro componentes: el primero es la parte de la volatilidad que permanece constante; el se- 
gundo se asocia con los rangos medios condicionales de los dos días inmediatos anteriores; un tercer componente tiene que ver con la contribución a la volatilidad por la información recibida en el corto plazo (rango observado del día inmediato anterior) y finalmente, como último componente, la variable Cant.Tit que resulta significativa además de tener una relación inversa.

De acuerdo con el número de supuestos que satisface cada uno de estos tres modelos, éste último cumple con todos; por tanto, podemos decir que para este modelo los MLE se comportan como insesgados, consistentes y eficientes. Además, se satisface que los errores se distribuyen como ruido blanco gaussiano.

Modelos ajustados con observaciones semanales, quincenales y mensuales

En algunos trabajos sobre el tema se esboza la idea de que si se toman observaciones más espaciadas (semanales, quincenales o mensuales) la distribución de los rendimientos se aproxima a una normal y, además, disminuye la persistencia entre observaciones sucesivas de la serie. Esto finalmente se traduce en que los modelos así estimados satisfacen más fácilmente los supuestos que se requieren. Incluso el modelo presentado por Chou (2005) con el S\&P500 toma el rango semanal y no diario.

Con el propósito de observar el impacto que tiene la separación entre observaciones en la determinación del modelo de rangos para AMX, L se incluyen los resultados:

\section{Cuadro 1}

Modelos ARMA estimados con rangos asociados a distintos periodos

\begin{tabular}{cccccc} 
& $\omega$ & $\alpha$ & $\beta$ & Persistencia & E(Y) \\
\hline Semanal & .066 & .350 & .406 & .756 & .270 \\
Quincenal & .097 & .182 & .667 & .849 & .642 \\
Mensual & .141 & .085 & .734 & .819 & .779
\end{tabular}

En el cuadro anterior aparecen los modelos ARMA $(1,1)$. En los tres casos se emplean estimadores MCO y se ve una reducción substancial en la persistencia con relación a la que muestran los modelos de los incisos V.1 y V.2. 
Es importante resaltar que en los tres casos, si bien los modelos cumplen con la mayoría de los requisitos, incluso con el hecho de que los residuales se comportan como ruido blanco, éstos no se comportan como ruido blanco gaussiano (figura 6). Ésta es la razón fundamental por la que se tiene que recurrir a QMLE.

En el cuadro 2 se presentan los resultados en la determinación del modelo ECARR a partir de QMLE con observaciones a intervalos cada vez más amplios:

\section{Cuadro 2}

Modelos GARCH estimados con rangos asociados a distintos periodos

\begin{tabular}{cccccc} 
& $\omega$ & $\alpha$ & $\beta$ & Persistencia & E(Y) \\
\hline Semanal & .016 & .287 & .471 & .758 & .066 \\
Quincenal & .012 & .181 & .691 & .872 & .013 \\
Mensual & .018 & .071 & .798 & .869 & .137
\end{tabular}

En ninguno de los tres casos la distribución de los errores es una exponencial unitaria como se supone en esta clase de modelos; no obstante, aquí también la persistencia se reduce substancialmente con relación a la que muestran los modelos V.1 y V.2.

\section{Conclusiones}

Obtener la mejor estimación de la volatilidad esperada condicional es un requisito indispensable en posteriores aplicaciones tales como la valuación de opciones, medición de riesgos y determinación del VAR. Contar con distintas alternativas de modelación del modelo CARR amplía el abanico de posibilidades y evita tener a la propuesta de Chou (2005) como única opción posible.

Con los resultados de este trabajo se observa que los dos mejores modelos se logran cuando se supera la limitante de que los errores se distribuyen de forma distinta a la normal. Este beneficio es aún mayor cuando se transforma el CARR en CARRX al incluir una variable explicativa adicional.

La cantidad de títulos negociados es una medida de la liquidez y de la forma como fluye la información relevante durante la jornada. Incluir esta variable en la estimación del modelo GARCHX de la serie de los rendimientos cierre/cierre reduce la per- 
sistencia y tiene un impacto inmediato sobre la volatilidad (Okan, Olgun y Takmaz, 2009). Una vez más este mismo efecto se replica al medir la volatilidad mediante la serie de los rangos de valores extremos y se construye el modelo CARRX.

Para el caso de AMX, L, este hecho queda evidenciado al incluir como variable exógena la cantidad de títulos negociados durante la sesión. Este resultado es fuertemente consistente con la hipótesis de arribo secuencial de la información.

En este trabajo también se ilustra que al determinar el rango esperado condicional a partir de observaciones más espaciadas (semanales, quincenales o mensuales), no se obtiene ninguna ventaja en cuanto a una mejor estimación del CARR.

De acuerdo con la revisión bibliográfica realizada, se puede afirmar que es muy escasa la producción de trabajos de econometría financiera que hacen referencia a títulos y mercados latinoamericanos. En su mayoría, los trabajos existentes analizan instrumentos norteamericanos, europeos y asiáticos pero en el caso particular de México la producción es muy escasa. Por tal razón, es indispensable promover y difundir la investigación financiera empleando este tipo de metodologías que sin duda contribuyen a una comprensión más precisa de las fortalezas y debilidades de los mercados y de los productos que ahí se negocian.

\section{Referencias}

Baklac1, H. F. y A. Kasman (2006). An Empirical Analysis of Trading Volume and Return Volatility Relationship in The Turkish Stock Market, Ege Akademik Bakis, Vol. 6, pp. 115-126.

Bollerslev, T. y Domowitz (1993), Trading Patterns and Prices in the Interbank Foreign Exchange Market, Journal of Finance, Vol.48, No.4, pp.1421-1443.

Chou, Heng-Chih y David Wang (2006). Using Conditional Autoregressive Range Model to Forecast Volatility of Stock Indices, Ming Chuan University, Taiwan; (c) Atlantis Press.

Chou, Yeutien R (2005). Forecasting Financial Volatilities whit Extreme Values: The Conditional Autoregressive Range (CARR) Model, Journal of Money Credit and Banking, Vol. 37, No. 3, 561-582. 
De Arce, Borda R (2001). 20 Años de Modelos ARCH: Una visión de conjunto de las distintas variantes de la familia. Universidad Autónoma de Madrid.

Díaz Mata, Alfredo (2008). Algunas consideraciones sobre el uso de técnicas fractales en el análisis del mercado accionario bursátil mexicano, Contaduría y Administración, Facultad de Contaduría y Administración, Universidad Nacional Autónoma de México, No. 224, enero-abril, México, 35-57.

De la Corte Barbás, Alejandro (2008). Las matemáticas en la economía financiera. Real Academia de las Ciencias Exactas, Físicas y Naturales de Madrid, Vol. 102, No. 1, pp 285-293.

Engle R. y A. Patton (2001). What Good is a Volatility Model. Quantitative Finance, Volume 1.

Engle R. (2002). New Frontiers for ARCH Models. Journal of Applied Econometrics. J. Appl. Econ. 17: 425-446.

Engle R, (2003). Riesgo y Volatilidad: Modelos Econométricos y Práctica Financiera. The Nobel Foundation. Revista Asturiana de Economía - RAE, No. 31 2004. (PP?)

Graham L. Giller (2005). A Generalized Error Distribution. Guiller Investments Research Note: 20031222/1, 1-7.

Jorion, Philippe (2004). Valor en riesgo. El nuevo paradigma para el control de riesgos con derivados. Limusa/Noriega Editores.

Malik, Magdon I. y Amir F. Atiya (2003). “A maximum likelihood to volatility estimation for a Brownian motion using the high, low, and close". Quantitative Finance, 1-9.

Miralles, Marcelo J. y J. Miralles Quirós (2007). “Análisis de la capacidad de predicción del S\&P 500 mediante estadísticos simétricos y asimétricos: Propuesta de una alternativa al modelo CARR".

Parkinson, M. (1980). "The Extreme Value Method for Estimating the Variance of the Rate of Return". The Journal of Business, Vol. 53, No. 1. 
Pacurar, María (2006). “Autoregressive Conditional Duration (ACD) Models in Finance: A survey of the Theorical and Empirical Literature". Dalhouse University. Canada.

Peiro Gimenez, A. (1992). "Distribución de los rendimientos de acciones". Estadística Española Vol. 34, No. 131.

Vuorenmaa Tommi A. (2006). "Q-Weibull Autoregressive Conditional Duration Model and threshold dependence". University of Helsinki, Ruesg and Hecer. Discussion Paper No. 117.

Tsay Ruey, S. Analysis of Financial Series, Wiley Interscence, 2005.

Wooldridge, Jeffrey M. (2001). "Applications of Generalized Method of Moments Estimation”. Journal of Economic Perspectives, Volume 15, No. 4, 87-100. 


\section{Apéndice}

\section{Figura 4}

Gráfica Q-Q comparando RPt con la lognormal

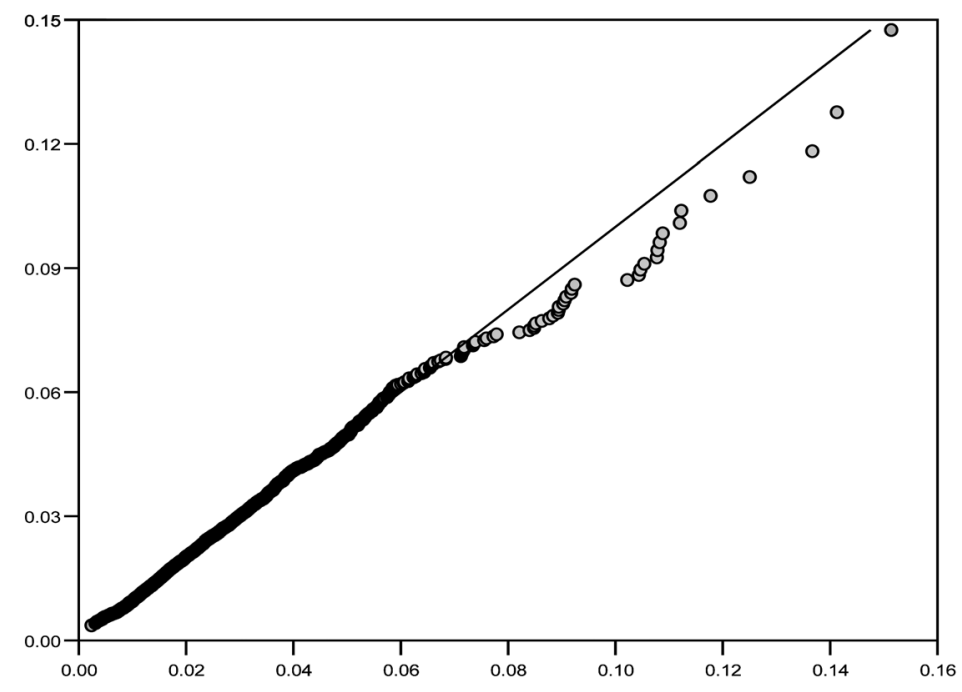

La distribución de frecuencias de los rangos se ajusta a una lognormal sin importar que éstos sean con observaciones diarias, semanales, quincenales o mensuales.

\section{Cuadro 3}

\section{Prueba asociada a los datos $\left\{\mathbf{R P}_{t}\right\}$}

Null Hypothesis: Rango Porcentual has a unit root

Exogenous: Constant

Bandwidth: 28 (Newey-West using Bartlett kernel)

\begin{tabular}{llll}
\hline \hline & Adj. t-Stat & Prob. $^{*}$ \\
\hline \hline Phillips-Perron test statistic & -39.06154 & 0.0000 \\
\hline Test critical values: & $1 \%$ level & -3.433338 & \\
& $5 \%$ level & -2.862746 & \\
& $10 \%$ level & -2.567458 & \\
\end{tabular}




\section{Cuadro 4}

\section{Modelo ARMA (1,1) estimado mediante MCO}

\begin{tabular}{lclll}
\hline \hline \multicolumn{1}{c}{ Variable } & Coefficient & Std. Error & t-Statistic & Prob. \\
\hline \hline C & 0.026700 & 0.001509 & 17.69698 & 0.0000 \\
MA(1) & 0.946445 & 0.009719 & 97.37670 & 0.0000 \\
\hline \hline R-squared & -0.720644 & 0.021187 & -34.01298 & 0.0000 \\
Adjusted R-squared & 0.345205 & Mean dependent var & 0.026922 \\
S.E. of regression & 0.344566 & S.D. dependent var & 0.016162 \\
Sum squared resid & 0.013084 & Akaike info criterion & -5.833348 \\
Log likelihood & 5990.932 & F-statistic & -5.825126 \\
Durbin-Watson stat & 1.883410 & Prob(F-statistic) & 540.3745 \\
\hline \hline Inverted AR Roots & .95 & & 0.000000 \\
Inverted MA Roots & .72 & & \\
\hline \hline
\end{tabular}

\section{Cuadro 5}

Prueba BDS asociada a los residuales del segundo modelo

BDS Test for RESID02

Sample: 2/07/2001 4/03/2009

Included observations: 2054

\begin{tabular}{|c|c|c|c|c|}
\hline Dimension & BDS Statistic & Std. Error & $\underline{\text { z-Statistic }}$ & Prob. \\
\hline 2 & 0.032212 & $\overline{0.001605}$ & $\overline{20.07433}$ & $\overline{0.0000}$ \\
\hline 3 & 0.056273 & 0.002548 & 22.08947 & 0.0000 \\
\hline 4 & 0.069859 & 0.003030 & 23.05699 & 0.0000 \\
\hline 5 & 0.074635 & 0.003154 & 23.66546 & 0.0000 \\
\hline 6 & 0.074350 & 0.003037 & 24.48006 & 0.0000 \\
\hline \multicolumn{2}{|c|}{ Raw epsilon } & 0.060731 & & \\
\hline \multicolumn{2}{|c|}{ Pairs within epsilon } & 2972944. & V-Statistic & 0.704670 \\
\hline \multicolumn{2}{|c|}{ Triples within epsilon } & $4.62 E+09$ & V-Statistic & 0.532914 \\
\hline
\end{tabular}


Cuadro 6

Modelo ECARR (1,1) estimado

\begin{tabular}{|c|c|c|c|c|}
\hline & Coefficient & Std. Error & z-Statistic & Prob. \\
\hline \multicolumn{5}{|c|}{ Variance Equation } \\
\hline C & 0.001725 & 0.000334 & 5.168076 & 0.0000 \\
\hline $\operatorname{RESID}(-1)^{\wedge} 2$ & 0.203032 & 0.018522 & 10.96175 & 0.0000 \\
\hline GARCH(-1) & 0.731576 & 0.025419 & 28.78120 & 0.0000 \\
\hline R-squared & -12.980341 & \multicolumn{2}{|c|}{ Mean dependent var } & 0.158128 \\
\hline Adjusted R-squared & -12.993973 & \multicolumn{2}{|c|}{ S.D. dependent var } & 0.043901 \\
\hline S.E. of regression & 0.164226 & \multicolumn{2}{|c|}{ Akaike info criterion } & -0.819245 \\
\hline Sum squared resid & 55.31577 & \multicolumn{2}{|c|}{ Schwarz criterion } & -0.811026 \\
\hline Log likelihood & 844.3647 & \multicolumn{2}{|c|}{ Durbin-Watson stat } & 0.074688 \\
\hline
\end{tabular}

Figura 5

Gráfica Q-Q comparando los residuales con la exponencial

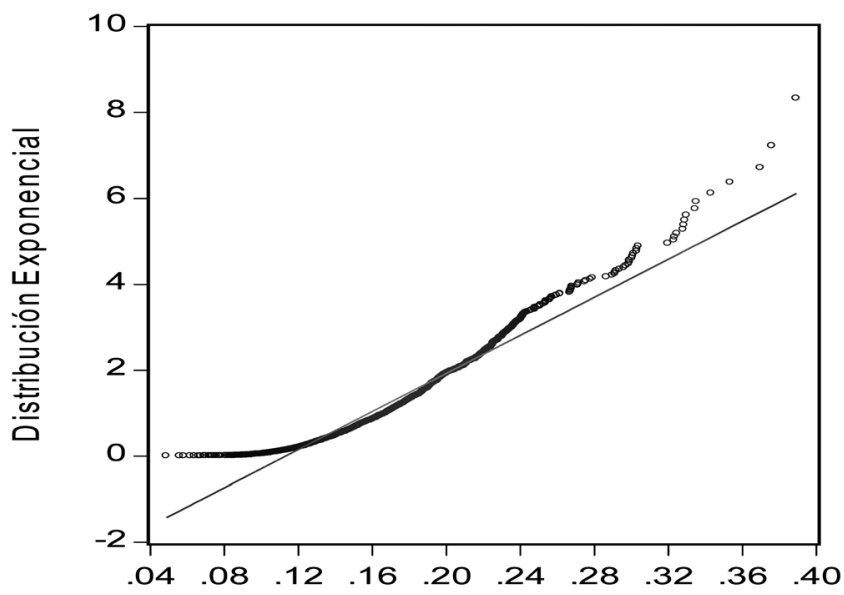




\section{Cuadro 7}

Modelo CARRX $(1,1)$ estimado

\begin{tabular}{lrllr}
\hline \hline & Coefficient & Std. Error & z-Statistic & Prob. \\
\hline \hline \multicolumn{5}{c}{ Variance Equation } \\
C & 0.026931 & 0.002929 & 9.195925 & 0.0000 \\
RESID(-1)^2 & 0.150000 & 0.053033 & 2.828420 & 0.0047 \\
GARCH(-1) & 0.600000 & 0.039068 & 15.35797 & 0.0000 \\
CANT_TIT & $-2.50 E-10$ & $1.65 E-12$ & -151.0816 & 0.0000 \\
\hline \hline & & & & \\
\hline \hline R-squared & -12.980341 & Mean dependent var & 0.158128 \\
Adjusted R-squared & -13.007633 & S.D. dependent var & 0.043901 \\
S.E. of regression & 0.164306 & Akaike info criterion & -0.549792 \\
Sum squared resid & 55.31577 & Schwarz criterion & -0.536093 \\
Log likelihood & 569.6366 & Durbin-Watson stat & 0.074688 \\
\hline \hline
\end{tabular}

\section{Figura 6}

Gráfica Q-Q de los residuales del ARMA con observaciones semanales

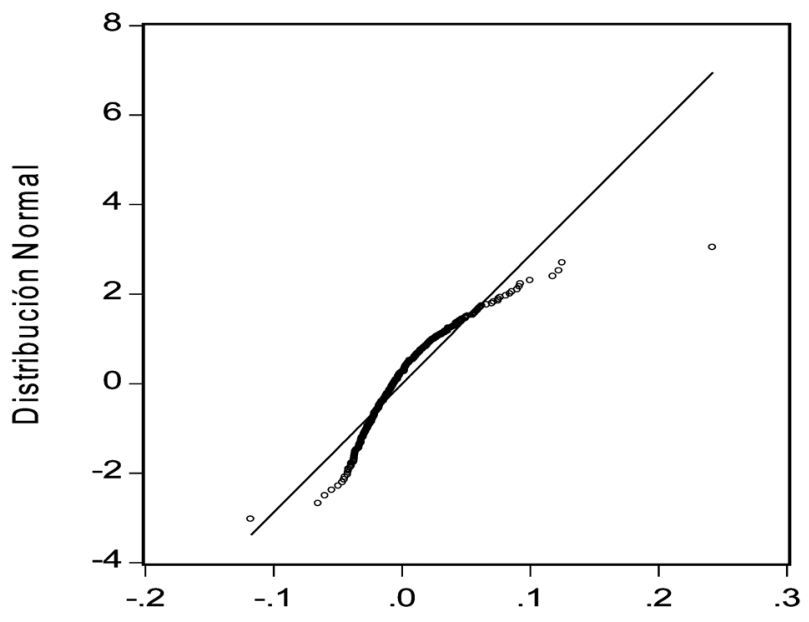

